\title{
Modulation of the velocity gradient tensor by concurrent large-scale velocity fluctuations in a turbulent mixing layer
}

\author{
By O.R.H. Buxton $\dagger$ \\ Department of Aeronautics, Imperial College London, London, SW7 2AZ, UK
}

(Received 22 June 2015)

The modulation of small-scale velocity and velocity gradient quantities by concurrent large-scale velocity fluctuations is observed by consideration of the Kullback-Leibler divergence. This is a measure that quantifies the loss of information in modelling a statistical distribution of small-scale quantities conditioned on concurrent positive large-scale fluctuations by that conditioned on negative large-scale fluctuations. It is observed that the small-scale turbulence is appreciably "rougher" when the concurrent large-scale fluctuation is positive in the low-speed side of a fully developed turbulent mixing layer which lends further evidence to the convective scale modulation argument of Buxton \& Ganapathisubramani (2014). The definition of the small scales is varied and regardless of whether the small-scale fluctuations are dominated by dissipation or have the characteristic features of inertial range turbulence they are shown to be modulated by the concurrent large-scale fluctuations. The modulation is observed to persist even when there is a large gap in wavenumber space between the small and large scales, although local maxima are observed at intermediate length scales that are significantly larger than the pre-defined small-scales. Finally, it is observed that the modulation of small-scale dissipation is greater than that for enstrophy with the modulation of the vortex stretching term, indicative of the interaction between strain-rate and rotation, being intermediate between the two.

\section{Introduction}

The study of Rao et al. (1971) first illustrated the coupling between large and small scales in a turbulent flow through investigation of the bursting phenomenon in a turbulent boundary layer. This has led to an increased interest in the coupling between these inner and outer scales in wall bounded flows in more recent years with Hutchins \& Marusic (2007) proposing a modulation of the small, near-wall structures by the larger, outer structures.

The comprehensive study of Bandyopadhyay \& Hussain (1984) was the first to extend this idea of an interaction between the large and small scales in a number of different shear flows, including both wall bounded and free shear flows. Through examination of short time correlations between the low pass filtered time series of data from hot wire experiments and the envelope of the small-scale (high pass filtered) component the authors were able to demonstrate a significant degree of coupling between the scales across all shear flows. The coupling between the scales was observed to be maximised when the high frequency and low frequency signals were concurrent. Mathis et al. (2013) laid the

$$
\dagger \text { corresponding author - o.buxton@imperial.ac.uk }
$$


theoretical framework for the "quasi-steady" description of the inner-outer modulation in turbulent boundary layers, further suggesting that concurrent modulation effects are of great significance in turbulent flows.

Whilst a large proportion of the work investigating scale interactions in turbulent flows has concentrated on wall bounded flows some significant progress has been made by looking at free shear flows through the prism of large eddy simulation sub-grid scale (SGS) models. For example, Meneveau (1994) took single point measurements in grid turbulence to compute joint moments between real (measured) SGS stresses and large-scale (filtered) velocity fluctuations. This was extended by O'Neil \& Meneveau (1997) who showed that large-scale organised structures within a turbulent free shear flow are shown to impact the statistical distribution of small-scale (SGS) velocity gradient quantities, such as the dissipation rate. The study of Buxton \& Ganapathisubramani (2014) presented evidence for the concurrent interaction between large-scale velocity fluctuations in a fully developed turbulent mixing layer and the "roughness" of the fine-scale turbulence. Due to intrinsic experimental uncertainties analogues to dissipation, namely $\epsilon \sim \nu\left(\frac{u_{S}}{\lambda}\right)^{2}$ and $\epsilon \sim \sigma_{u_{S}}$, in which $\lambda$ is the Taylor microscale and $\sigma_{u_{S}}$ is the variance of the small-scale fluctuations, were used to identify the modulation of small-scale dissipation by large-scale fluctuations. In this manuscript we thus choose to examine a direct numerical simulation (DNS) dataset in order to directly observe the modulation of dissipation by large-scale velocity fluctuations without recourse to dissipation analogues.

\section{Data}

The data are identical to that used by Buxton et al. (2011) in the developed, far field region of a turbulent planar mixing layer that closely matches the experimental dataset of Buxton \& Ganapathisubramani (2014). The mixing layer is produced by means of a DNS of two flows of different free stream velocities, $U_{1}$ and $U_{2}$ in the ratio $U_{1} / U_{2}=2$, either side of a splitter plate of thickness $h$ to which a wedge of angle $4^{\circ}$ is appended to produce a sharp trailing edge. The computational domain $\left(L_{x} \times L_{y} \times L_{z}\right)=(230.4 h \times 48 h \times 28.8 h)$ is discretised onto a Cartesian mesh that is stretched in the cross stream $(y)$ direction of $(2049 \times 513 \times 256)$ mesh nodes. The stretching of the mesh in the cross stream direction leads to a minimal mesh size of $\Delta y \approx 0.03 h$. The time step, $\Delta t=0.05 h / U_{c}$, in which $U_{c}=\left(U_{1}+U_{2}\right) / 2$ is the mean convection velocity, is low enough to satisfy the CourantFriedrichs-Lewy condition, ensuring temporal stability of the simulation.

The code "incompact3d" is used to solve the incompressible non-dimensionalised NavierStokes equations. Details on the numerical schemes for this code can be found in Laizet \& Lamballais (2009). The boundary conditions are inflow/outflow in the streamwise direction (velocity boundary conditions of the Dirichlet type), free slip in the cross-stream direction at $y= \pm L_{y} / 2$ and periodic in the spanwise direction at $z= \pm L_{z} / 2$. The pressure mesh is staggered from the velocity mesh to avoid spurious pressure oscillations. Using the concept of modified wavenumber, the divergence free condition is ensured up to the machine accuracy.

A sub domain that consisted of the final $301(\times 512 \times 256)$ mesh nodes in the streamwise, $x$, direction was isolated at three time steps that were sufficiently well spaced in time to ensure statistical independence from one another and stored. The sub domain is in the far field of the mixing layer in which the turbulence is fully developed with self-similar mean velocity profiles throughout, with all subsequent data and analyses presented in this manuscript coming from this sub domain. A threshold based on enstrophy was devised to discriminate between the turbulent and potential flow within the sub domain. 
Only data points for which $\omega^{2}>0.025\left\langle\omega^{2}\right\rangle(t)$ are included in the statistics presented in this manuscript, in which $\left\langle\omega^{2}\right\rangle(t)$ is the mean enstrophy for each stored time step, accounting for some $30 \%$ of the original data. Within this region of the flow the centreline Reynolds number based on the Taylor micro-scale is $R e_{\lambda} \approx 220$ which approximates the experimental mixing layer data of Buxton \& Ganapathisubramani (2014) $\left(R e_{\lambda} \approx 260\right.$ at the centre-line).

\section{Filtering}

In order to observe the modulation of the small-scale velocity gradient phenomena by the concurrent large-scale velocity fluctuations it is necessary to filter the data. Throughout this manuscript the velocity field is filtered with a sharp spectral cut-off filter, implemented in three dimensions such that

$$
\boldsymbol{u}_{\boldsymbol{S}}=\mathcal{F}^{-1}\left\{\hat{\boldsymbol{U}}_{\boldsymbol{S}}(\boldsymbol{\kappa})\right\} \quad \text { where } \hat{\boldsymbol{U}}_{\boldsymbol{S}}(\boldsymbol{\kappa})=\left\{\begin{array}{rcc}
0 & \forall & |\boldsymbol{\kappa}|<\frac{1}{\Lambda}_{S} \\
\mathcal{F}\{u\} & \forall & |\boldsymbol{\kappa}| \geq \frac{1}{\Lambda}{ }_{S}
\end{array}\right.
$$

in which $\mathcal{F}$ denotes the three-dimensional Fourier transform operator, $\boldsymbol{\kappa}$ is a threedimensional wavenumber vector and $\Lambda_{S}$ is the filter length that defines the small scales. N.B. the definition of the wavenumber neglects the factor of $2 \pi$ for simplicity. Since the original simulation was run on a gird that was stretched in the $y$ direction the data were interpolated onto a uniform grid in which $\Delta y=\Delta x(=\Delta z)$ prior to the implementation of the sharp spectral cut-off filter. The filter lengths are visualised in figure 1 , which shows the dissipation spectrum for the central part of the mixing layer in which the enstrophy threshold is met. The dashed lines represent filter lengths of $\Lambda_{S}=\lambda, 2 \lambda, 3 \lambda$ and $4 \lambda$, where $\lambda$ is the Taylor micro-scale, and thus $\boldsymbol{u}_{\boldsymbol{S}}$ contains content to the right hand side of these dashed lines. The large-scale velocity field is similarly defined

$$
\boldsymbol{u}_{\boldsymbol{L}}=\mathcal{F}^{-1}\left\{\hat{\boldsymbol{U}}_{\boldsymbol{L}}(\boldsymbol{\kappa})\right\} \quad \text { where } \hat{\boldsymbol{U}}_{\boldsymbol{L}}(\boldsymbol{\kappa})=\left\{\begin{array}{rcc}
\mathcal{F}\{u\} & \forall & |\boldsymbol{\kappa}| \leq \frac{1}{\Lambda}{ }_{L} \\
0 & \forall & |\boldsymbol{\kappa}|>\frac{1}{\Lambda_{L}}
\end{array} .\right.
$$

N.B. in the subsequent analysis $\Lambda_{S}$ does not necessarily equal $\Lambda_{L}$ and thus modulations across a "gap" in wavenumber space are presented. The small-scale velocity gradient field, $\frac{\partial \boldsymbol{u}_{\boldsymbol{S}}}{\partial \boldsymbol{x}}(\boldsymbol{x}, t)$, is then numerically computed by fitting sixth order Lagrange interpolating polynomials through the small-scale velocity field in the $O x, O y$ and $O z$ directions and calculating the tangent to these polynomials at $\boldsymbol{x}$.

With regards to the velocity gradient tensor, the generalised topology of a turbulent flow can be shown to depend solely on the second and third invariants, $Q$ and $R$ respectively, of this tensor (Chong et al. 1990). Further, the joint probability density function ( $p d f$ ) between $Q$ and $R$ is known to take a characteristic "tear-drop" shape for a number of fully developed turbulent flows such that it is considered a universal aspect of fine-scale turbulence. The discriminant, $\Delta=Q^{3}+(27 / 4) R^{2}$ for an incompressible flow, separates purely real (straining) from complex (swirling) states of the flow (Perry \& Chong 1994) and is known to act as an attractor leading to the so-called "Viellefosse tail" (Vieillefosse 1982) in the lower right hand quadrant. The effect of filtering the velocity fields at various $\Lambda_{S}$ is thus illustrated in figure 2 , which shows the joint $p d f$ s between $Q$ and $R$ for the small-scale velocity gradient fields filtered at various length scales. The classical "tear-drop" shape can be seen to develop as the filter length is increased from $\Lambda_{S}=\lambda$ (a) to $\Lambda_{S}=4 \lambda(\mathrm{d})$. In particular the filling out of the "Vieillefosse tail" and the upper left quadrant (defined by $\Delta>0 ; R<0$ ) which Buxton \& Ganapathisubramani (2010) showed to be where enstrophy amplification $\left(\Omega=\omega_{i} s_{i j} \omega_{j}>0\right)$ is dominant is observed 


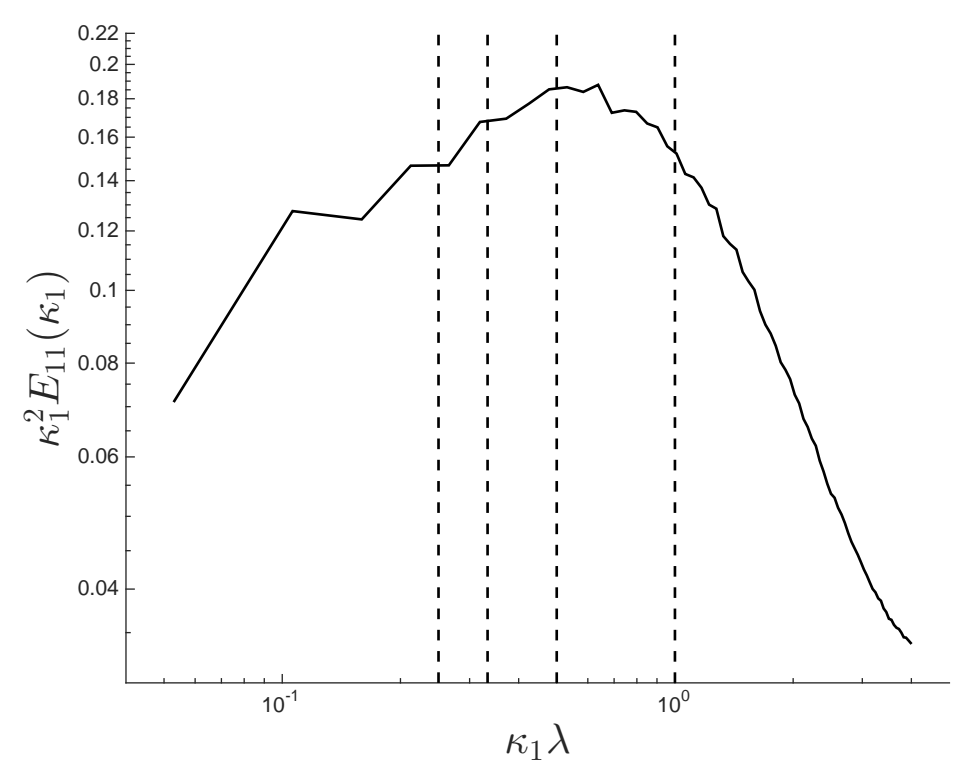

FIgURE 1. One-dimensional dissipation spectrum for the region of the mixing layer fulfilling the minimum enstrophy threshold. From left to right the dashed lines mark the cut-off filter lengths of $\kappa_{1} \lambda=\frac{1}{4}, \kappa_{1} \lambda=\frac{1}{3}, \kappa_{1} \lambda=\frac{1}{2}$ and $\kappa_{1} \lambda=1$ respectively.

as $\Lambda_{S}$ is increased. Contrastingly, for the smallest filter length, $\Lambda_{S}=\lambda$, which can be seen to correspond to a wavenumber that is greater than that for the peak of the dissipation spectrum in figure 1, a classical "tear-drop" shape is not present. Neither the "Vieillefosse tail" nor the upper left hand quadrant are observed to be well developed, and its shape resembles that produced from within the turbulence production region of the turbulence generating grids of Gomes-Fernandes et al. (2014) and the transitional boundary layer data of Elsinga et al. (2012) (private communication). In both of these flows there are few length scales present which is mimicked in the case of $\Lambda_{S}=\lambda$ of figure 2(a). The "tear-drop" shape of the $Q-R$ joint $p d f$ thus appears to be driven by the presence of a broad range of scales in the inertial range as opposed to simply the dissipative scales. Qualitatively, this can be linked to the conditional mean trajectories of $Q$ and $R$ presented in a turbulent boundary layer from the study of Atkinson et al. (2012). It is shown that in the viscous and buffer layers, in which the range of scales is small, there is an attraction to smaller gradients at the origin $(Q=R=0)$ in time, whereas in the log and outer layers these trajectories "fill out" the upper left hand quadrant and are attracted to the "Vieillefosse tail". This is explained by a smaller contribution from the viscous diffusion term in the dynamics of the velocity gradient tensor.

\section{Results and discussion}

Buxton \& Ganapathisubramani (2014) proposed a convective mechanism for the modulation of the "roughness" of the small-scale turbulence by the large-scale velocity fluctuations. For a developed turbulent free shear flow the peak ensemble averaged Reynolds stresses are observed on the centreline and thus a positive cross-stream $(v)$ fluctuation will on average convect a fluid element of "rougher" turbulence towards the high speed side of the mixing layer. Due to the non-negativity of the mean turbulent kinetic energy 


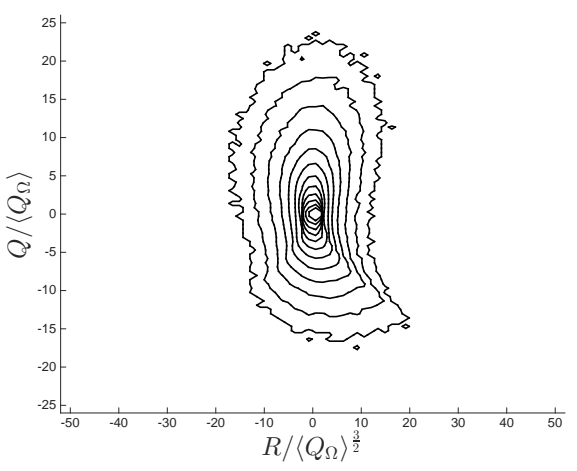

(a)

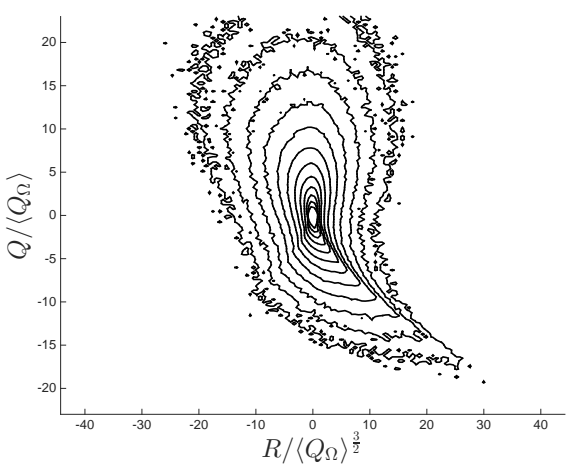

(c)

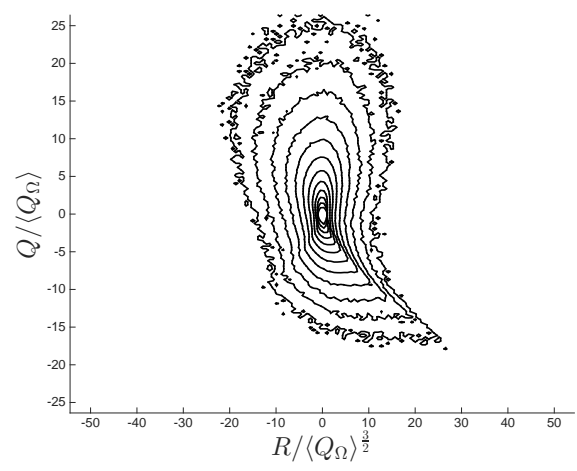

(b)

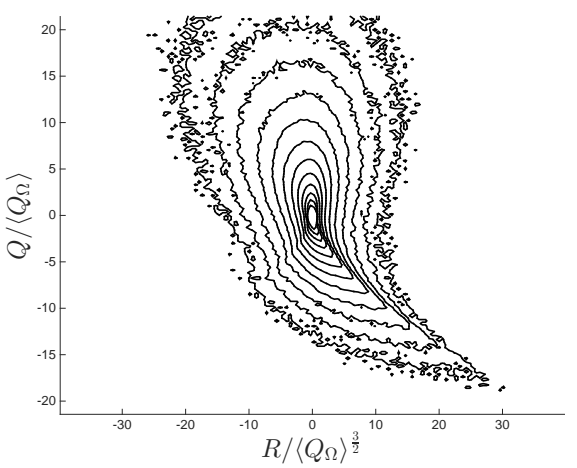

(d)

FiguRE 2. Joint $p d f$ s between $Q$ and $R$ for the high pass filtered (in wavenumber space) velocity gradient fields in which $\Lambda_{S}=$ (a) $\lambda$, (b) $2 \lambda$, (c) $3 \lambda$ and (d) $4 \lambda$. Contour levels are logarithmically spaced from $10^{-5.6}$ to $10^{-1}$.

(TKE) production term for a nominally two-dimensional free shear flow, $\mathcal{P}=-\langle u v\rangle \frac{\partial \bar{U}}{\partial y}$, a positive $v$ fluctuation is inversely correlated with a negative $u$ fluctuation, explaining their finding that "rougher" small-scale turbulence is found concurrently to negative $u$ fluctuations. Additional evidence for this convective modulation hypothesis is presented in figure 3(a), which shows the pdfs of $u_{S}$ conditioned on the sign of $u_{L}$ for the case in which $\Lambda_{S}=\Lambda_{L}=2 \lambda$ in the low-speed side of the mixing layer, i.e. $y<0$. It can be seen that the $p d f$ conditioned on $u_{L} \geq 0$ has a lower modal peak and broader tails indicating an increase in small-scale turbulent activity concurrent to positive $u_{L}$ fluctuations. These are correlated to a negative (downward) $v_{L}$ fluctuation, which is the opposite finding to Buxton \& Ganapathisubramani (2014) for the high-speed side of the mixing layer, as required by the proposed convective mechanism. N.B. all subsequent results are derived from the low-speed side of the mixing layer for consistency with figure 3(a).

Figures $3(\mathrm{~b})$ to $(\mathrm{d})$ show the $p d f \mathrm{~s}$ of small-scale dissipation $\left(\epsilon_{S}\right)$, enstrophy $\left(\omega_{S}{ }^{2}\right)$ and enstrophy amplification term $\left(\Omega_{S}=\omega_{i} s_{i j} \omega_{j}\right)$, in which $s_{i j}$ represents the fluctuating strain-rate tensor $s_{i j}=\frac{1}{2}\left(\frac{\partial u_{S, i}}{\partial x_{j}}+\frac{\partial u_{S, j}}{\partial x_{i}}\right)$, conditioned on the sign of $u_{L}$. Whereas Buxton \& Ganapathisubramani (2014) were able to show that the small-scale turbulence is modulated to be "rougher" via a dissipation analogue it is clear that the $p d f \mathrm{~s} \epsilon_{S}, \omega_{S}{ }^{2}$ 


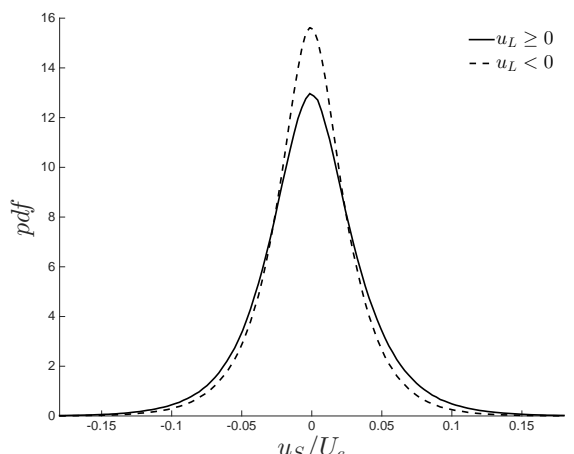

(a)

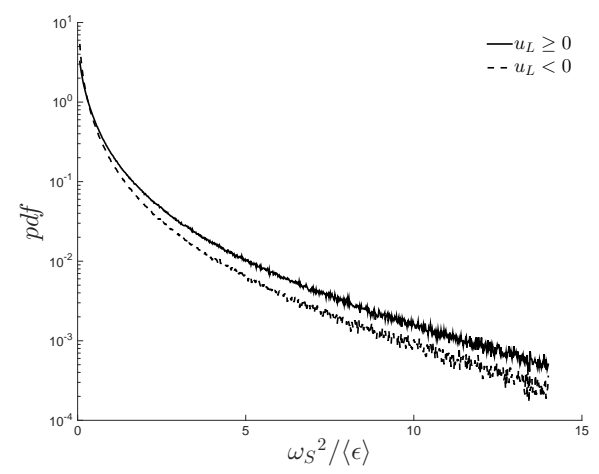

(c)

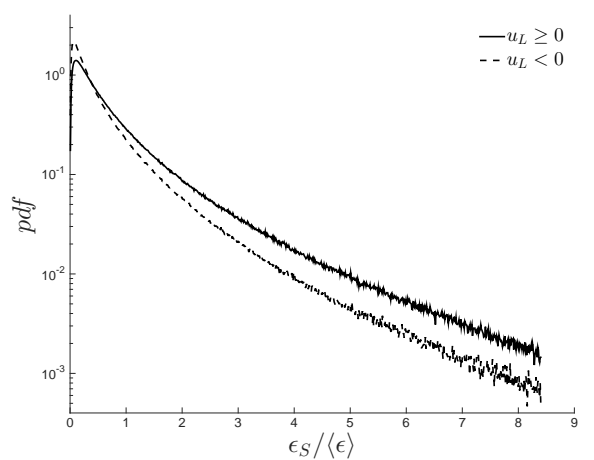

(b)

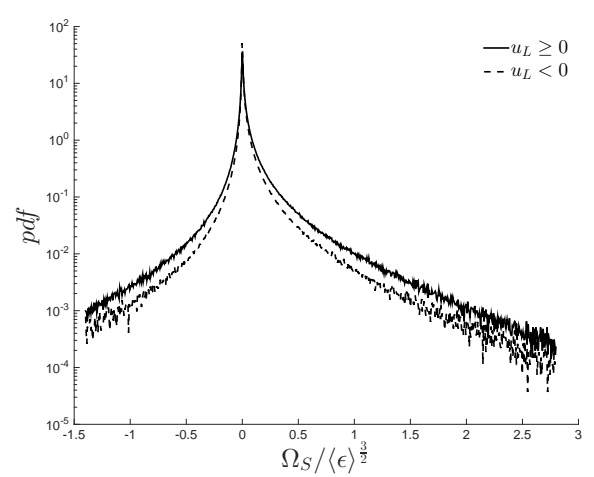

(d)

Figure 3. pdfs of (a) $u_{S}$ fluctuations, (b) dissipation, (c) enstrophy and (d) the enstrophy amplification term conditioned on $u_{L} \geq 0$ (solid lines) and $u_{L}<0$ (dashed lines) for $\Lambda_{S}=\Lambda_{L}=2 \lambda$.

and $\Omega_{S}$ all have more extensive tails when conditioned on positive $u_{L}$ than negative $u_{L}$. Interestingly it can be observed that both the enstrophy attenuating $\left(\Omega_{S}<0\right)$ and the enstrophy amplifying $\left(\Omega_{S}>0\right)$ tails are enhanced by concurrent positive $u_{L}$ fluctuations.

The difference between the two conditional $p d f \mathrm{~s}$ in figure 3 can be quantified by means of the Kullback-Leibler divergence (KLD) (Kullback \& Leibler 1951). The KLD is a nonnegative, non-symmetric measurement of the difference between two probability density functions and is defined as

$$
D_{K L}(A \| B)=\int_{-\infty}^{\infty} \ln \left[\frac{a(X)}{b(X)}\right] a(X) \mathrm{d} X
$$

in which $a(X)$ and $b(X)$ are probability density functions of a fluctuating variable $X$ $\left(D_{K L}(A \| B)=0\right.$ only if the distributions $A$ and $B$ are identical). The KLD originates from information theory and is asymmetric such that $D_{K L}(A \| B) \neq D_{K L}(B \| A)$. $D_{K L}(A \| B)$ can be thought of as the loss of information/power as a hypothesised distribution $A$ is misspecified as $B$ (Eguchi \& Copas 2006). We may thus quantify the difference between the $p d f \mathrm{~s}$ of figure 3 conditioned on the sign of the large-scale velocity fluctuations, and hence the magnitude of the scale modulation, through the KLD. The advantage of using the KLD to quantify the difference between the two conditional $p d f \mathrm{~s}$ is that it is based around the $\log$ likelihood ratio $\ln [a(X) / b(X)]$. As can be seen in figure 3 the high magnitude, intermittent dissipation/enstrophy events are more than 


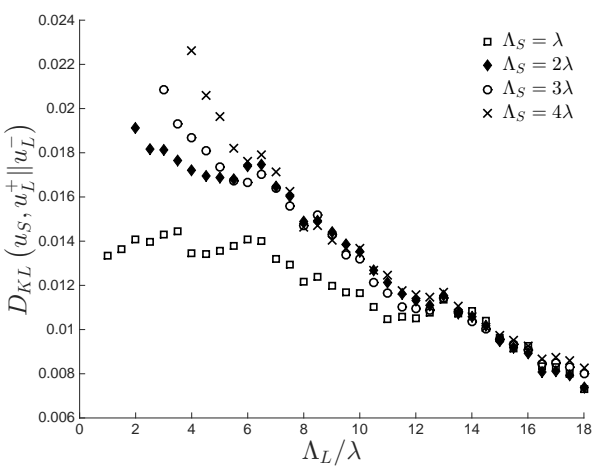

(a)

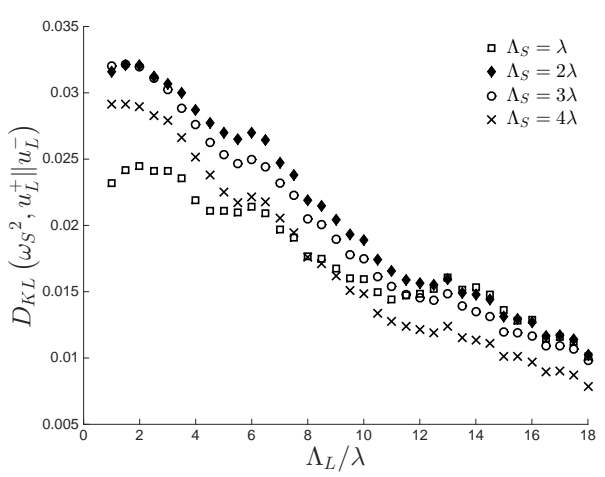

(c)

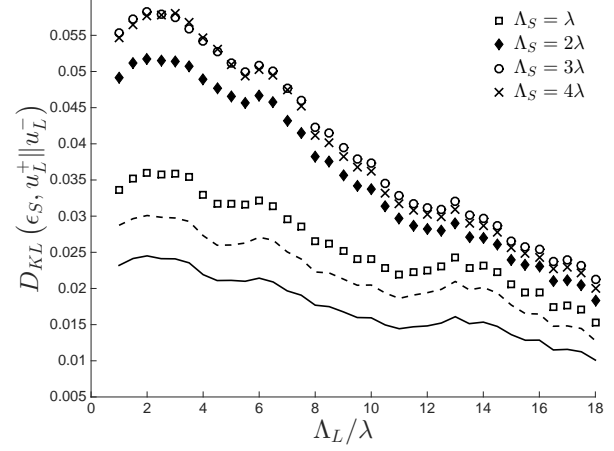

(b)

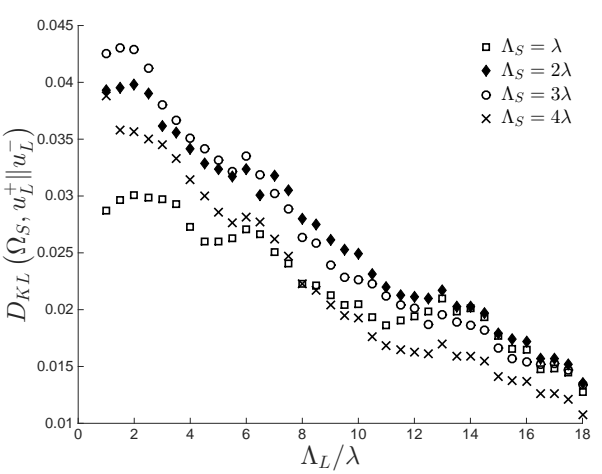

(d)

Figure 4. Kullback-Leibler divergence, $D_{K L}\left(X_{S}, u_{L}^{+} \| u_{L}^{-}\right)$for $X_{S}=u_{S}$ (a), $\epsilon_{S}$ (b), $\omega_{S}^{2}$ (c) and $\Omega_{S}$ (d) for $1 \leq \Lambda_{L} / \lambda \leq 18$. N.B. $D_{K L}\left(\omega_{S}^{2}, u_{L}^{+} \| u_{L}^{-}\right)$and $D_{K L}\left(\Omega_{S}, u_{L}^{+} \| u_{L}^{-}\right)$for $\Lambda_{S}=\lambda$ are plotted as a solid line and dashed line respectively in (b) to indicate their relative magnitudes.

three orders of magnitude less probable than the modal events and it is important to ensure that this is factored into the quantification of the scale modulation, particularly so for higher Reynolds number turbulent flows in which the intermittency is larger. The notation $D_{K L}\left(X_{S}, u_{L}^{+} \| u_{L}^{-}\right)$denotes the KLD for the $p d f$ of quantity $X_{S}$ (which may be $u_{S}, \epsilon_{S}, \omega_{S}^{2}, \Omega_{S}$ etc.) conditioned on positive large-scale velocity fluctuations to that conditioned on negative large-scale velocity fluctuations. Thus, a larger-value of $D_{K L}\left(X_{S}, u_{L}^{+} \| u_{L}^{-}\right)$is indicative of a more significant modulation effect and will, in general, be a function of both $\Lambda_{S}$ and $\Lambda_{L}$. In practise, of course, an integration from $-\infty$ to $\infty$ is not possible, hence the $p d f$ s of figure 3 are truncated, neglecting $<1 \%$ of the data at the extremities of the tails. The two discrete $p d f$ s are then sampled at the same values of $X_{S}$ and thus the integral of equation 4.1 is evaluated numerically over the truncated range of $X_{S} .800$ bins were used to formulate the $p d f \mathrm{~s}$, which was observed to make the computation of $D_{K L}\left(X_{S}, u_{L}^{+} \| u_{L}^{-}\right)$insensitive to the resolution of the $p d f$ s/statistical convergence, and the truncation was chosen such that the computation of $D_{K L}\left(X_{S}, u_{L}^{+} \| u_{L}^{-}\right)$ was also observed to be insensitive to this choice. This validation is not presented for brevity.

Figure 4 presents $D_{K L}\left(X_{S}, u_{L}^{+} \| u_{L}^{-}\right)$for $X_{S}=u_{S}(\mathrm{a}), \epsilon_{S}$ (b), $\omega_{S}^{2}$ (c) and $\Omega_{S}$ (d) for $1 \leq \Lambda_{L} / \lambda \leq 18$. This range is limited to preserve the Nyquist sampling theorem in the cross-stream, $y$, direction for the implementation of the sharp spectral cut-off filter. Thus 
it can be seen that there is a difference between the $p d f$ s conditioned on positive largescale fluctuations and negative large-scale fluctuations for all four small-scale quantities presented in figure 4 up to $\Lambda_{L}=18 \lambda$ and for all four $\Lambda_{S}$ tested. This is indicative of a scale modulation that is non-local in wavenumber space in which even very large-scale (purely inertial) fluctuations modulate the concurrent small-scale behaviour. This modulation decays with $\Lambda_{L}$, from a peak value at around $\Lambda_{L} \approx 3 \lambda$, regardless of $\Lambda_{S}$, but is present nonetheless at large values of $\Lambda_{L}$.

Since the constituent $p d f$ s are conditioned on the large-scale velocity fluctuations figure 4(a) is presented only for the cases of $\Lambda_{L}>\Lambda_{S}$. The modulation effect is seen to be greater as $\Lambda_{S}$ is increased for smaller values of $\Lambda_{L}$. This is intuitive, since one is effectively closing the "gap" in wavenumber space by increasing $\Lambda_{S}$ for a given $\Lambda_{L}$. However, for $\Lambda_{S} \geq 2 \lambda$ it can be observed that the KLD plots effectively merge at a value of $\Lambda_{L} \approx 6.5 \lambda$ before collapsing. This scale modulation by large/very large scales is thus observed to depend not upon the "gap" in wavenumber space but on the physical size of the large/very large scales themselves. Thus, there is an apparent physical significance attributable to the length scale $\Lambda \approx 6.5 \lambda$ after which "local" (in wavenumber space) effects become important in the modulation of small velocity fluctuations by concurrent large ones, which shall be discussed in the paragraph below.

The KLD for $\Lambda_{S}=\lambda$ displays qualitatively different behaviour to the other three, with a much flatter decay than for the other three over low and intermediate values of $\Lambda_{L}$. Figure 2 shows that when $\Lambda_{S}=\lambda$ the exaggerated "tear-drop" shape of the $Q-R$ joint $p d f$ is not observed, whereas it is (to varying extents) for $\Lambda_{L} \geq 2 \lambda$. These velocity fluctuations can thus be considered to consist almost entirely of dissipative scale fluctuations. The merger with the other $D_{K L}\left(u_{S}, u_{L}^{+} \| u_{L}^{-}\right)$curves is now observed to take place at $\Lambda_{L} \approx 13 \lambda$, which is close to a sub-harmonic value to that for the other merger point. It is additionally observed that for low values of $\Lambda_{L}$ there are two distinct regions, from $\lambda \lesssim \Lambda_{L} \lesssim 3.5 \lambda$ and $4 \lambda \lesssim \Lambda_{L} \lesssim 6.5 \lambda$ over which the KLD is observed to increase with $\Lambda_{L}$ before then falling away rapidly. The second of these rapid drop-offs coincides with the merger point at $\Lambda_{L} \approx 6.5 \lambda$. It is difficult to apportion a physical significance to a length scale of $13 \lambda$ (and harmonic at $6.5 \lambda$ ). Whilst an insufficient number of time steps of the data were stored to accurately compute the integral length scale at this point of the flow a coarse estimate based on an exponential fit to the longitudinal correlation function suggests that $L \approx 13 \lambda$ is in the right "ball park". This is in agreement with the data of Buxton \& Ganapathisubramani (2014) when adjusted for the lower $R e_{\lambda}$. It thus appears that the scale modulation effect for velocity fluctuations may be driven by the integral scale streamwise rollers that are present in a turbulent mixing layer, linking the convective scale interaction mechanism with the eddy structure of the flow, although this conclusion should be treated with some caution.

Some other general observations from the figure may be made. Firstly, it can be seen that the magnitude of the modulation is greater for the small-scale velocity gradient quantities (b)-(d) than for the small-scale velocity fluctuations (a) for smaller values of $\Lambda_{L}$. This is, however, observed to converge as $\Lambda_{L} \rightarrow 18 \lambda$ as the modulation effect diminishes across the large gap in wavenumber space. It should be noted that the $D_{K L}\left(X_{S}, u_{L}^{+} \| u_{L}^{-}\right)$ curves for various $\Lambda_{S}$ do not collapse for velocity gradient quantities, unlike those for velocity fluctuations. Additionally, the decay in $D_{K L}\left(X_{S}, u_{L}^{+} \| u_{L}^{-}\right)$with $\Lambda_{L}$ is not observed to be smooth but has three small, but distinct local peaks at $\Lambda_{L} \approx 3.5 \lambda, \lambda_{L} \approx 6.5 \lambda$ and $\Lambda_{L} \approx 13 \lambda$. These correspond to the merger points for the $D_{K L}\left(u_{S}, u_{L}^{+} \| u_{L}^{-}\right)$curves of figure 4(a) and are linked to the streamwise integral length scale. Finally, it can be seen for all cases that there is a significant increase in the scale modulation effect as $\Lambda_{S}$ is increased from $\lambda$ to $2 \lambda$. This corresponds to the cut-off wavenumber moving from below 
the peak of the dissipation spectrum to the peak value in figure 1 . As $\Lambda_{S}$ is increased further the modulation effect varies according to $X_{S}$ which is discussed further below.

The largest modulation effect is present for $X_{S}=\epsilon_{S}$. Figure 4(b) shows the KLD for the dissipation, enstrophy and enstrophy amplification term for $\Lambda_{S}=\lambda$ on the same axes for comparison. It can be seen that $D_{K L}\left(\epsilon_{S}, u_{L}^{+} \| u_{L}^{-}\right)$is consistently around 1.5 times that of $D_{K L}\left(\omega_{S}^{2}, u_{L}^{+} \| u_{L}^{-}\right)$, the solid line of figure 4(b), across the entire range of $\Lambda_{L}$. The scale modulation of rotation is thus significantly less than that for strain-rate, i.e. the symmetric part of the velocity gradient tensor is more sensitive to concurrent velocity fluctuations than the skew-symmetric part. This is reinforced by the observation that $D_{K L}\left(\Omega_{S}, u_{L}^{+} \| u_{L}^{-}\right)$, the dashed line of figure $4(\mathrm{~b})$, is intermediate between that for dissipation and enstrophy. von Kármán (1937) first identified $\Omega_{S}=\omega_{i} s_{i j} \omega_{j}$ as the inviscid source/sink term in the enstrophy equation as the interaction between rotation and strain-rate. It is thus revealed that the scale modulation of this quantity is intermediate between that for rotation and strain-rate.

Additionally the increase in modulation effect, for $\Lambda_{L} \lesssim 10 \lambda$ at least, as $\Lambda_{S}$ is increased from $\lambda$ to $2 \lambda$ is greater for dissipation (b) than for enstrophy (c). This may be linked to the finding of figure 2, that as the definition of $\Lambda_{S}$ is broadened the "Vieillefosse tail" is the region that is significantly extended, which is strain-rate (dissipation) dominated. However, whilst the modulation effect is observed to increase further as $\Lambda_{S}$ is increased from $2 \lambda$ to $3 \lambda$ for dissipation (b) this is not the case for enstrophy (c). At the largest value of $\Lambda_{S}=4 \lambda$ the modulation effect is similar to that for $\Lambda_{S}=3 \lambda$ for dissipation but has decayed significantly for enstrophy. In particular, it can be seen that at lower values of $\Lambda_{L} D_{K L}\left(\omega_{S}^{2}, u_{L}^{+} \| u_{L}^{-}\right)$computed from $\Lambda_{S}=4 \lambda$ is lower than both $\Lambda_{S}=3 \lambda$ and $\Lambda_{S}=2 \lambda$, whilst at the highest values of $\Lambda_{L}$ the modulation effect is smallest for $\Lambda_{S}=4 \lambda$ than any other values. Exactly the same trend is followed in (d) for $D_{K L}\left(\Omega_{S}, u_{L}^{+} \| u_{L}^{-}\right)$. Contrastingly, the scale modulation for dissipation increases up to $\Lambda_{S}=3 \lambda$ and remains unchanged for $\Lambda_{S}=4 \lambda$. This is in contrast to the modulation of the velocity fluctuations (figure $4(\mathrm{a})$ ) in which (at low $\Lambda_{L}$ ) the modulation effect is observed to increase monotonically as $\Lambda_{S}$ is increased.

\section{Conclusions}

The hypothesised convective scale modulation mechanism presented in Buxton \& Ganapathisubramani (2014) is lent further credence in this manuscript. A concurrent scale modulation of the small-scale velocity fluctuations, and importantly velocity gradient quantities, is observed in which positive $u_{L}$ fluctuations contain "rougher" small-scale turbulence with more intermittent dissipation, enstrophy and enstrophy amplification in the low-speed side of a mixing layer. This scale modulation is observed to occur when the small-scales, defined by a sharp cut-off wavenumber, consist entirely of dissipative motions as well as inertial motions. It is visualised in figure 5, which illustrates the "wormlike" structures of high enstrophy, which are widely reported in the literature (e.g. Kerr 1985 ), concurrent to isosurfaces of $u_{L} / U_{c}= \pm 0.1$. The positive velocity fluctuations (red isosurfaces) are clearly more densely populated with the high enstrophy "worms" than then negative fluctuations (blue isosurfaces).

The scale modulation peaks when the cut-off is close to the peak of the dissipation spectrum in which the characteristic "tear-drop" shaped joint $p d f$ between the second and third invariants of the velocity gradient tensor begins to take shape, namely the extended "Vieillefosse tail" and enhanced contribution from the primarily enstrophy amplifying sector. The "tear-drop" shape itself is only produced when a significant range of scales is present, beyond merely the dissipative range. The scale modulation effect is 


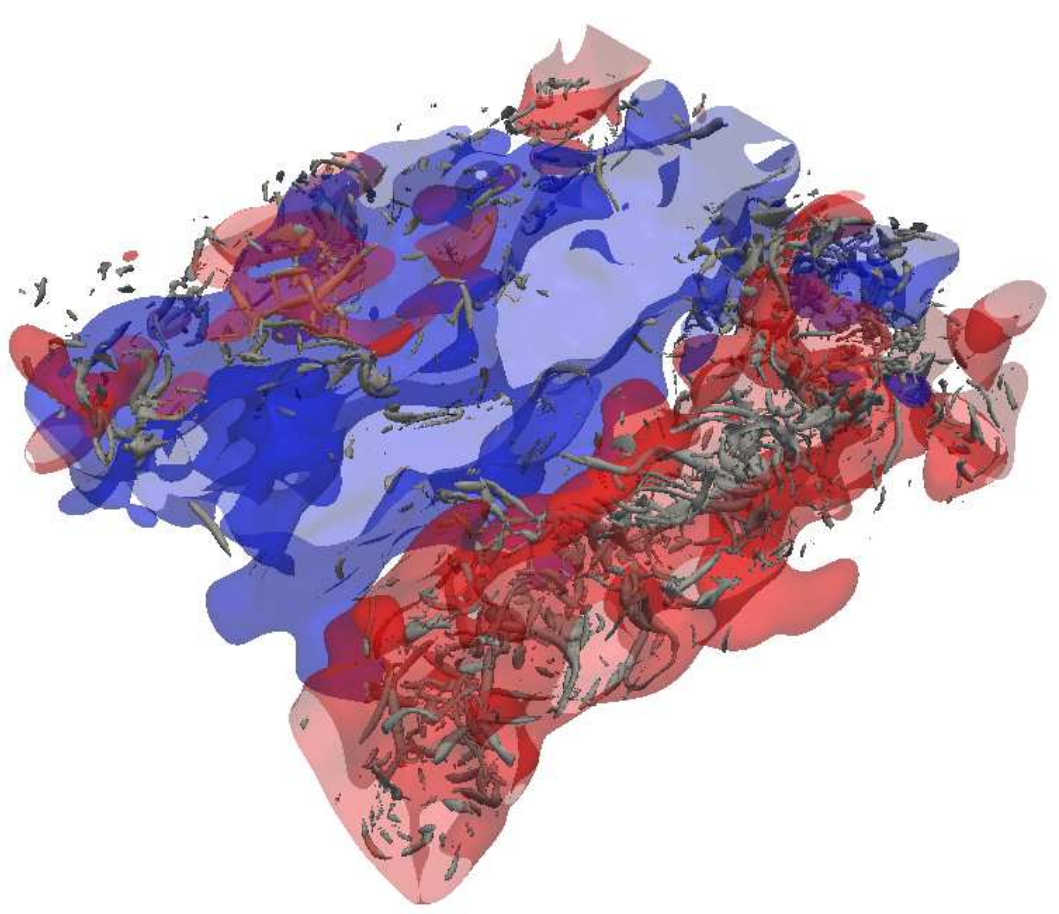

Figure 5. Instantaneous visualisation of high enstrophy $\left(\omega_{S}{ }^{2}\right)$ "worms" concurrent to $u_{L} / U_{c}=0.1$ (red) and $u_{L} / U_{c}=-0.1$ (blue) isosurfaces for the low-speed side of the mixing layer. In this case $\Lambda_{S}=\Lambda_{L}=3 \lambda$.

shown to be non-local in the sense that very large scale velocity fluctuations alter the distribution of concurrent small-scale velocity and velocity gradient quantities across a large "gap" in wavenumber space. The modulation of small-scale velocity fluctuations by very large scales collapses below a certain wavenumber definition for these large scales, whereas this collapse is not observed for velocity gradient quantities. The sign of the large-scale velocity fluctuations is also observed to modulate the small-scale velocity gradient quantities, with a peak modulation length scale observed to be $\Lambda_{L} \approx 3 \lambda$, regardless of how the small scales are defined. Further, the modulation of strain (dissipation) is observed to be more significant than that for rotation (enstrophy), with vortex stretching intermediate between the two as illustrated in figure 4(b). As the definition of the small scales is broadened, including a greater contribution to the total dissipation, the modulation effect increases up to a point. This is observed to lie between $3 \lambda$ and $4 \lambda$ for dissipation and somewhat smaller than this for enstrophy and enstrophy amplification, after which the modulation effect diminishes. This is not observed for the modulation of small-scale velocity fluctuations which is observed to increase monotonically (over the range of $\Lambda_{S}$ tested) as the definition of the small scales is broadened. The modulation effect is observed to spike when the large-scales are harmonics of the integral length scale, linking this mechanism to the large-scale rollers that are present in turbulent shear flows. It can thus be postulated that the convective mechanism for scale interaction is driven by the large-scale engulfment of regions of high small-scale activity, close to the peak Reynolds stress location, which are then transported in the cross-stream direction.

The author would like to acknowledge EPSRC for the provision of computational time 
on HECToR through the Resource Allocation Panel, Dr. Sylvain Laizet for his role in simulating the data and Mr. Mel Chamand for fruitful discussions on scale interactions.

\section{REFERENCES}

Atkinson, C., Chumakov, S., Bermejo-Moreno, I. \& Soria, J. 2012 Lagrangian evolution of the invariants of the velocity gradient tensor in a turbulent boundary layer. Physics of Fluids (1994-present) 24 (10), - .

Bandyopadhyay, P. \& Hussain, A. 1984 The coupling between scales in shear flows. Phys. Fluids 27 (9), 2221-2228.

Buxton, O. \& Ganapathisubramani, B. 2010 Amplification of enstrophy in the far field of an axisymmetric turbulent jet. J. Fluid Mech. 651, 483-502.

Buxton, O., Laizet, S. \& Ganapathisubramani, B. 2011 The interaction between strainrate and rotation in shear flow turbulence from inertial range to dissipative length scales. Phys. Fluids 23 (061704), 1-4.

Buxton, O. R. H. \& Ganapathisubramani, B. 2014 Concurrent scale interactions in the farfield of a turbulent mixing layer. Physics of Fluids (1994-present) 26 (12), 125106:1-19.

Chong, M., Perry, A. \& CAntwell, B. 1990 A general classification of three-dimensional flow fields. Phys. Fluids A 2 (5), 765-777.

Eguchi, S. \& Copas, J. 2006 Interpreting Kullback-Leibler divergence with the NeymanPearson lemma. Journal of Multivariate Analysis 97 (9), 2034-2040.

Elsinga, G., Poelma, C., Schröder, A., Geisler, R., Scarano, F. \& Westerweel, J. 2012 Tracking of vortices in a turbulent boundary layer. J. Fluid Mech. 697, 273-295.

Gomes-Fernandes, R., Ganapathisubramani, B. \& Vassilicos, J. 2014 Evolution of the velocity-gradient tensor in a spatially developing turbulent flow. Journal of Fluid Mechanics 756, 252-292.

Hutchins, N. \& Marusic, I. 2007 Large-scale influences in near-wall turbulence. Phil. Trans. R. Soc. Lond. 365, 647-664.

vON KÁRmán, T. 1937 The fundamentals of the statistical theory of turbulence. Journal of the Aeronautical Sciences 4 (4), 131-138.

KERR, R. 1985 Higher-order derivative correlations and the alignment of small-scale structures in isotropic numerical turbulence. J. Fluid Mech. 153, 31-58.

Kullback, S. \& Leibler, R. A. 1951 On information and sufficiency. The Annals of Mathematical Statistics 22 (1), 79-86.

Laizet, S. \& LAmballais, E. 2009 High-order compact schemes for incompressible flows: A simple and efficient method with quasi-spectral accuracy. J. Comp. Phys. 228 (16), 59896015.

Mathis, R., Marusic, I., Chernyshenko, S. \& Hutchins, N. 2013 Estimating wall-shearstress fluctuations given an outer region input. Journal of Fluid Mechanics 715, 163-180.

Meneveau, C. 1994 Statistics of turbulence subgridscale stresses: Necessary conditions and experimental tests. Physics of Fluids (1994-present) 6 (2), 815-833.

O'Neil, J. \& Meneveau, C. 1997 Subgrid-scale stresses and their modelling in a turbulent plane wake. Journal of Fluid Mechanics 349, 253-293.

Perry, A. \& Chong, M. 1994 Topology of flow patterns in vortex motions and turbulence. Appl. Sci. Res. 53, 357-374.

RaO, K., Narasimha, R. \& Narayanan, M. 1971 The "bursting" phenomenon in a turbulent boundary layer. J. Fluid Mech. 48, 339-352.

Vieillefosse, P. 1982 Local interaction between vorticity and shear in a perfect incompressible fluid. J. Phys. (Paris) 43 (6), 837-842. 